\title{
Techniques to accelerate convergence of stress-controlled molecular dynamics simulations of dislocation motion
}

\author{
David Cereceda J. Manuel Perlado Jaime Marian
}

\begin{abstract}
A B S T R A C T
Dislocation mobility - the relation between applied stress and dislocation velocity-is an important property to model the mechanical behavior of structural materials. These mobilities reflect the interaction between the dislocation core and the host lattice and, thus, atomistic resolution is required to capture its details. Because the mobility function is multiparametric, its computation is often highly demanding in terms of computational requirements. Optimizing how tractions are applied can be greatly advantageous in accelerating convergence and reducing the overall computational cost of the simulations. In this paper we perform molecular dynamics simulations of $1 / 2\langle 111\rangle$ screw dislocation motion in tungsten using step and linear time functions for applying external stress. We find that linear functions over time scales of the order of $10-20$ ps reduce fluctuations and speed up convergence to the steady-state velocity value by up to a factor of two.
\end{abstract}

\section{Introduction}

In bcc materials such as tungsten (W), the plastic behavior is controlled by screw dislocations with Burgers vector $\mathbf{b}=1 / 2$ $\langle 111\rangle$ due to their low mobility and non-planar core structure [1-3]. At low homologous temperatures $1 / 2\langle 111\rangle$ screw dislocations typically glide on $\{110\}$ or $\{112\}$ type planes by thermallyactivated nucleation and propagation of kink pairs. At a critical stress known as Peierls stress, $\sigma_{P}$, lattice friction is suppressed and dislocation motion is characterized by interaction with lattice phonons. Because dislocation motion essentially reflects the interaction of the core with the host lattice, atomistic resolution is needed, and thus methods capable of providing it such as molecular dynamics (MD). The so-called mobility function may thus display as many dependencies as there are variables that define the atomistic system, e.g. lattice structure, dislocation type, direction and plane of motion, atomic composition, etc. This multiparametric nature of the mobility function makes it computationally intensive, even when using MD simulations based on semi-empirical interatomic potentials. In addition, the thermally-activated regime is governed by rare-event dynamics, which adds also to the computational cost of atomistic simulations.

MD simulations of dislocation motion are defined by the triad formed by the line, plane normal, and glide directions [4,5]. The line direction $\xi$ must reflect the character of the dislocation, e.g.
〈111) for screw dislocations in bcc metals. The plane normal direction $\mathbf{n}$ describes the slip plane, e.g. $\langle 110\rangle$ or $\langle 112\rangle$. The glide direction $\mathbf{m}$ can be arbitrary, although it is common to take $\mathbf{m}=\boldsymbol{\xi} \times \mathbf{n}$ so that orthorhombic simulation cells can be used. A single dislocation in such a cell breaks the periodicity of the box along the normal direction resulting in surfaces with normal vector $\mathbf{n}$ of finite dimensions. Typically, stress or strain rate boundary conditions are imposed on these surfaces to drive dislocation motion. Both methods are valid and should converge in the infinite time and length limits. However, unlike strain-controlled simulations, which prescribe the dislocation velocity (from $v=\dot{\varepsilon} / \rho b$, where $\dot{\varepsilon}$ is the strain rate, $\rho$ the dislocation density, and $b \equiv\|b\|$ ), stress-controlled simulations are independent on the size of the computational cell. In this paper we focus on strategies about how to impart the stress such that the fidelity of the simulations is optimized while keeping the computational cost manageable. We try both step and linear time stress functions and assess their effect on the simulations output via clearly defined metrics such as stress oscillation amplitudes and dislocation velocities.

\section{Methodology}

A typical setup for stress-controlled screw dislocation simulations is shown in Fig. 1.

The principal axes $x, y$, and $z$ of the MD cell were oriented along

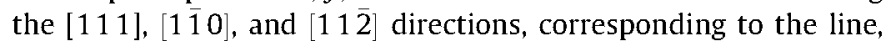
glide and normal directions respectively. The screw dislocation is 


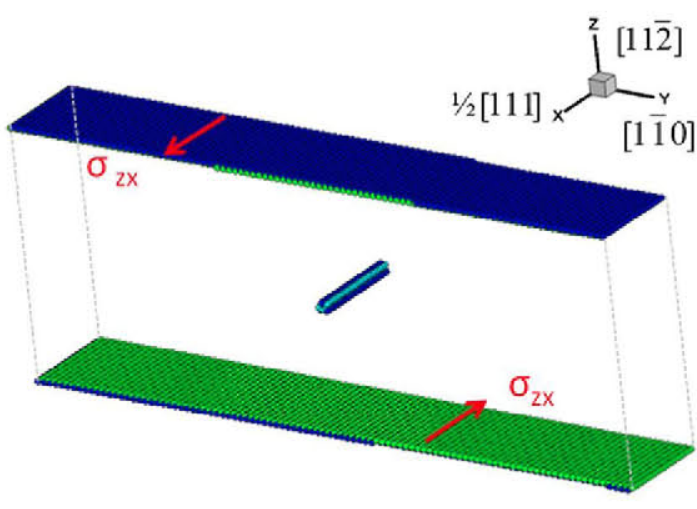

Fig. 1. MD simulation setup.

generated at the center of the box by using the isotropic elastic displacement solution [4].

Periodic boundary conditions are imposed along the $x$ and $y$ directions, whereas traction surfaces bound the system along the $z$ direction. Stress is applied on these surfaces via surface forces that satisfy $F=\sigma A$, where $\sigma$ is the desired stress and $A=L_{x} L_{y}$ is the exposed area defined by the dimensions along $x$ and $y$. For the orientation shown in Fig. 1, the stress component that creates a Peach-Kohler force in the glide direction is $\sigma_{z x}$. The traction $F$ is distributed evenly among one or several layers of atoms adjacent to the surface. Atoms in these layers are held rigid with respect to one another and are not subjected to MD.

The dimensions of the simulation box were chosen to maximize the physical fidelity of the simulations while keeping the computational cost manageable. First, the dislocation line length $L_{x}$ must be sufficiently long to support a kink-pair mechanism of motion under the proper conditions [6]. Second, the dimension along the glide direction $L_{y}$ was chosen such that the heat generated by the mechanical work during dislocation glide was dissipated before sequential dislocation passages through the periodic boundary. Finally, $L_{z}$ was set so as to keep image forces small around the glide plane. More details about these optimization strategies have been given by Gilbert et al. [7]. Of course, these strategies only set generic guidelines and the specific dimensions that satisfy them are potential dependent. Here we have used a modified embeddedatom method (MEAM) potential parameterized for $\mathrm{W}$ that predicts the symmetric correct core structure at $0 \mathrm{~K}$ and a Peierls stress of $3.2 \mathrm{GPa}$ [8]. After extensive numerical tests using this interatomic potential, we arrived at $L_{x}=5.48, L_{y}=44.7$, and $L_{z}=19.4 \mathrm{~nm}$.

Prior to the application of stress, the computational cell is equilibrated at the desired temperature in the microcanonical ensemble. Once stress is applied, the bulk region atoms are evolved in the $n p T$ ensemble. In the next section we discuss the effects of using different time functions for the applied stress. All MD simulations were performed using the massively parallel code LAMMPS [9] in a temperature range of $300-2100 \mathrm{~K}$, and a stress range of 200-2000 MPa.

\section{Results}

\subsection{Step functions}

A step function consists of a transition from 0 to $\sigma_{z x}$ from one MD time step to the next. This sudden application of stress results in elastic waves generated at the system boundaries that travel at the transversal speed of sound $\left(c_{t}=2890 \mathrm{~ms}^{-1}\right.$ for this potential). When these waves reach the opposite end of the computational cell, they reflect and travel back across the sample with reversed sign. In this fashion, every time either a positive or a negative step wave reaches the dislocation (situated at the center of the box), the dislocation will feel forward or arrest forces depending on the sign of traveling wave. This method is commonplace and has been widely used in the literature [7,10-12]. Its effects are shown in Fig. 2, where the dislocation displacement is plotted against time for a simulation at $1500 \mathrm{~K}$ and $1100 \mathrm{MPa}$. The figure shows a succession of steps resembling a stairway. The sloped part of the steps corresponds to exposure to positive elastic waves, whereas the 'flat' part results from the cancellation of the acting positive wave with a reverberating negative wave. A simple calculation shows that the duration of each step is of the order of $L_{z} / c_{t} \approx 7 \mathrm{ps}$.

To support this argument, we also plot in Fig. 2 the $\sigma_{z x}$ component of the virial stress with time. As shown, the shear stress wave oscillates around the prescribed value of applied stress. Overlaying $\sigma_{z x}$ to the dislocation displacement leads to two interesting observations. First, the period of the elastic wave is coincident with the duration of one step. Second, the stress wave amplitude reaches its maxima at the midpoint flat section of each step. These points correspond to the instances at which positive elastic waves traverse the dislocation glide plane. Conversely, the minima are attained when negative waves reach the glide plane. Traveling waves are gradually attenuated due to scattering processes as seen by the decrease in the amplitude of the displayed oscillations with time. It is expected that, as these oscillations are suppressed, the system reaches a state of homogeneous shear stress and the displacement-time curves become perfectly linear.

\subsection{Linear functions}

An alternative to the sudden application of stress is the use of some monotonic time function. For simplicity, here we explore linear functions noting that any other higher order function could be equally acceptable. In this fashion, we 'ramp up' the applied stress from zero to its prescribed value, $\sigma_{0}$, and start measuring dislocation displacement precisely at that time. The linear function is also characterized by the time duration of the ramp, $\theta: \sigma(t)=\frac{t}{\theta} \sigma_{0}$. Once the desired stress level is achieved, it is maintained for the remainder of the simulation. The results for the linear stress function case are also shown in Fig. 2. The figure shows data for a simulation where a slope of $\theta=20$ ps was used. As the figure shows, the amplitude of the oscillations is much reduced and the smoothness of the displacement-time curve is increased. More importantly, the overall slope of the displacement-time curves, i.e. the dislocation velocity, appears rather different at plain sight.

To further study the effect of the time constant on the shape of the displacement-time curves, we have carried out simulations in

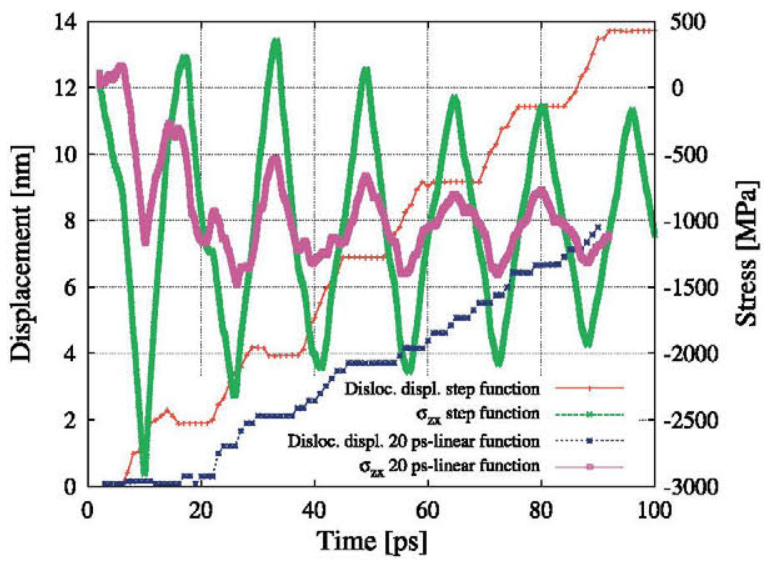

Fig. 2. Screw dislocation position as a function of time for a simulation at $T=1500 \mathrm{~K}, \sigma=1100 \mathrm{MPa}$ both for the case of a step function and a linear function with a slope of $20 \mathrm{ps}$. 


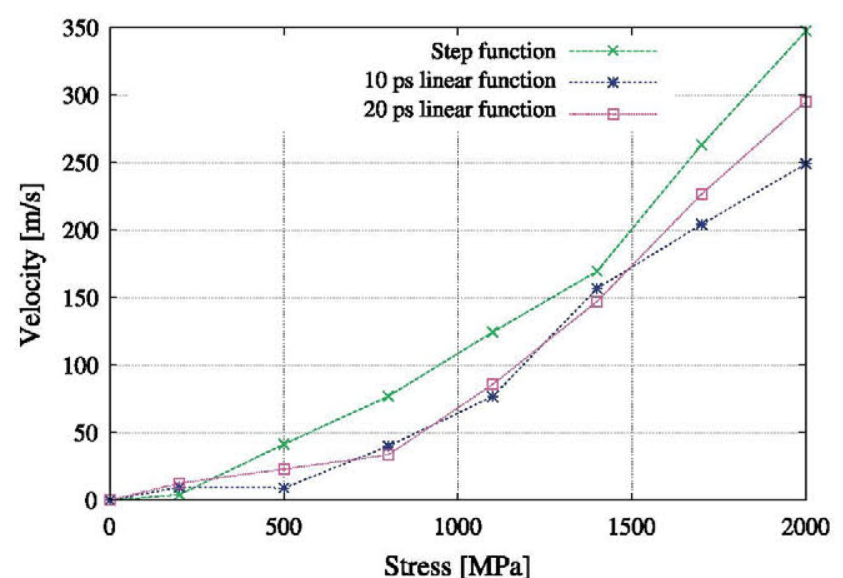

Fig. 3. Screw dislocation velocity at $1200 \mathrm{~K}$ using step function, linear function with $\theta=10 \mathrm{ps}$ and linear function with $\theta=20$ ps.

the 200-2000 MPa stress range every $300 \mathrm{MPa}$ using a step function and two linear functions with time constants of $\theta=10$ and $20 \mathrm{ps}$. We have found that the differences between these two are practically negligible. This is further confirmed by looking at the resulting velocities in each case, obtained as the derivatives of least-squares linear fits to the displacement-time data. These are plotted in Fig. 3.

The figure clearly shows that, except perhaps at $2000 \mathrm{MPa}$, using a linear function with 10 or 20 ps has not noticeable impact on the dislocation velocities. More worrisome is the fact that, as Fig. 2 shows, 100 ps may not sufficient to obtain converged results using a stress step. In the next section we explore the conditions under which step functions may be used safely on the basis of convergence with the linear function calculations.

\subsection{Long term convergence of mobility simulations}

As it was shown in Fig. 2, stress wave reflections are gradually dampened. The question that logically follows is at which point do fluctuations become of the same order as those that appear in simulations using linear stress functions. To this end, in Fig. 4 we extend step and linear function simulations up to 300 ps to study the convergence of stress fluctuations in each case. Also plotted is the exponential decay function that envelops the maxima of the oscillations in the step function simulations. This function is proportional to $e^{-0.02 t}$, i.e. defined by a time constant of $\tau \approx 50 \mathrm{ps}^{1}$.

Indeed, the figure shows that oscillations fall to within $3 \%$ of the steady state value (which is the range of the oscillations in the linear function case) after 185 ps of simulation, i.e. both approaches converge after a time of $3.5 \tau$. Furthermore, the slope of the displacement-time curves becomes virtually equivalent. The figure essentially suggests that spending 20 ps to build up shear stress prior to measuring velocities results in a net 165 ps savings of simulated time. This is a consideration to keep in mind when computational resources are limited.

\subsection{Screw dislocation mobility}

To emphasize the difference between 100 -ps simulations using step and linear functions to apply external stress, we plot in Fig. 5 the normalized difference in velocity from both approaches. The plotted values correspond to the ratio $\left(v_{s}-v_{l} / v_{l}\right)$, where $v_{s}$ and $v_{l}$

\footnotetext{
${ }^{1}$ The complete function is: $\sigma(t)=1606 e^{-0.02 t}-1387(\mathrm{MPa})$. The independent constant is indicative of the applied stress, while the exponential factor represents the
} mean lifetime $\tau$.

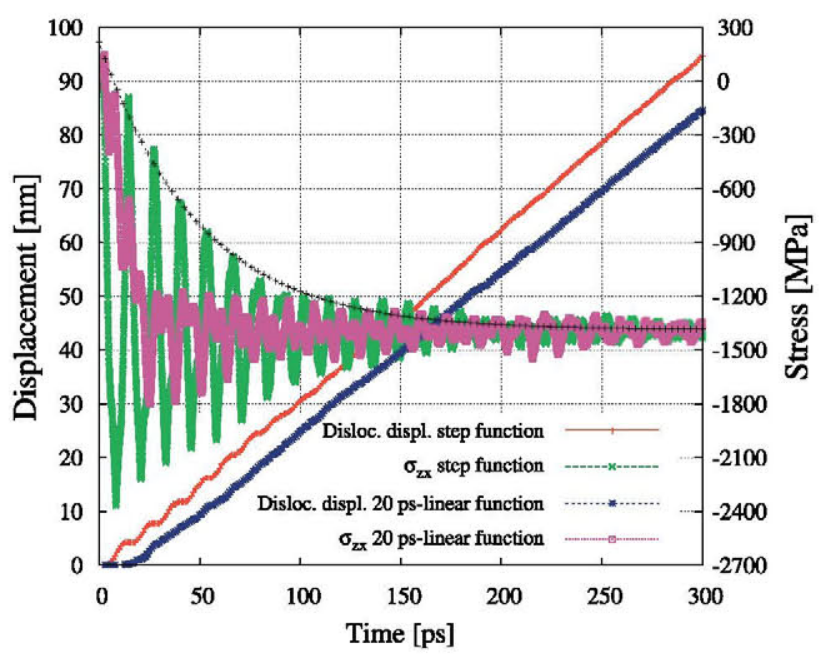

Fig. 4. Screw dislocation position as a function of time during a 300-ps simulation at $600 \mathrm{~K}$ and $1400 \mathrm{MPa}$. Also shown is the evolution of the $\sigma_{z x}$ component of the virial with time.

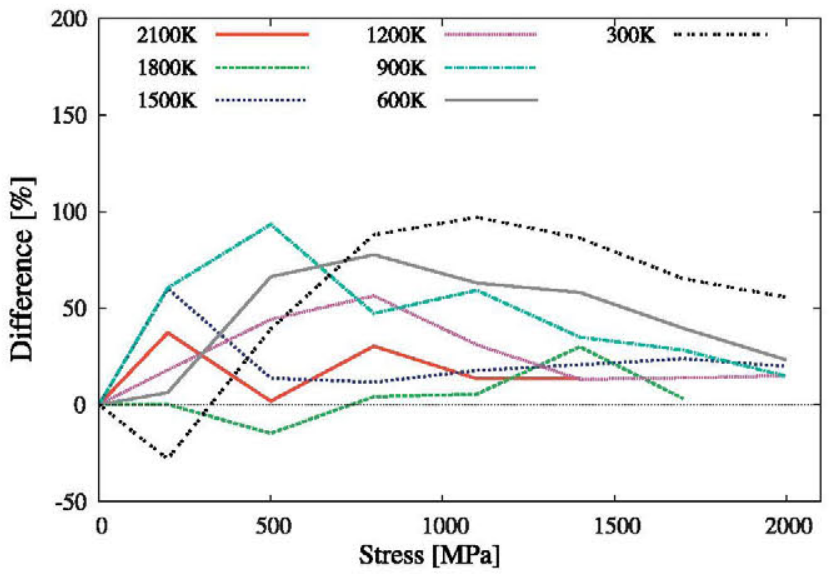

Fig. 5. Percentage difference in screw dislocation velocity between step and linear stress function simulations. The data are plotted as a function of temperature and stress and the difference is computed as $\left(v_{s}-v_{l} / v_{l}\right)$.

are the dislocation velocities obtained using step and linear stress functions. Interestingly, at and above $1200 \mathrm{~K}$, the differences in dislocation velocities are only slight, whereas at lower temperatures the differences are more pronounced. This is likely related to the fact that fluctuations are scattered more efficiently via interactions with lattice phonons at high temperature. Generally speaking, the dislocation velocities for the linear function case are lower under the same conditions of temperature and applied stress.

The potential employed here displays a Peierls stress of $3.2 \mathrm{GPa}$, which means that all the calculations performed here up to $2.0 \mathrm{GPa}$ fall within the thermally-activated regime of motion, governed by kink pair nucleation and propagation. It is not the objective of this paper, however, to investigate the details of that mechanism, which we leave for a future study [13].

\section{Discussion and conclusions}

The final objective in the application of the boundary conditions is to maximize the use of computational resources while ensuring that the desired observables are calculated with the highest degree of physical fidelity. Here we have seen that simple 'ramps' as short as $10 \mathrm{ps}$ may be sufficient to dampen stress oscillations to a level 
that requires nearly 200 ps to reach when stress is applied as a sudden step. It is not clear how other non-linear, albeit still smooth, functions will perform, although it is to be expected that they will behave no worse than the linear ones tried here.

Dislocation mobility calculations are computationally intensive and highly multiparametric, that is, many variables may need to be explored to have a minimally acceptable characterization of the mobility dependencies. Under these circumstances, even small CPU savings per parametric space point may result in better usage of computational resources.

We conclude by noting that, although interesting in themselves, the mobility data and a discussion of their validity are not the main subjects of this paper. The full analysis of the calculations shown in Fig. 5 will be carried out in a future publication.

\section{Acknowledgements}

We thank Dr. Max Victoria for useful suggestions and motivating discussions. This work performed under the auspices of the US Department of Energy by Lawrence Livermore National Laboratory under Contract DE-AC52-07NA27344 and the 7th Framework Programme (FP7) with the project: HiPER, European High Power Laser
Energy Research Facility (ESFRI), Grant Agreement No. 211737. We specifically acknowledge the $\mathrm{PhD}$ program support from Universidad Politecnica de Madrid.

\section{References}

[1] V. Vitek, Philosophical Magazine 84 (2004) 415-428.

2] W. Cai, V.V. Bulatov, J. Chang, J. Li, S. Yip, Dislocation core effects on mobility, in: Dislocations in Solids, Elsevier, pp. 1-80 (Chapter 64).

[3] A. Hartmaier, P. Gumbsch, Physical Review B 71 (2005) 024108.

[4] D. Hull, D.J. Bacon, Introduction to Dislocations, Butterworth-Heinemann, Oxford, 2001.

[5] V.V. Bulatov, W. Cai, Computer Simulations of Dislocations, Oxford University Press, 2006.

[6] J. Marian, W. Cai, V.V. Bilatov, Nature Materials 3 (2004) 158-163.

[7] M.R. Gilbert, S. Queyreau, J. Marian, Physical Review B 84 (2011) 174103.

[8] T. Lenosky, B. Sadigh, E. Alonso, V. Bulatov, T. de la Rubia, J. Kim, A. Voter, J. Kress, Modelling and Simulation in Materials Science and Engineering 8 (2000) $825-841$.

[9] S. Plimpton, Journal of Computational Physics 117 (1995) 1-19.

[10] J. Chaussidon, M. Fivel, D. Rodney, Acta Materialia 54 (2006) 3407-3416.

[11] R. Gröger et al., Acta Materialia 56 (2008) 5401-5411.

[12] P.A. Gordon, T. Neeraj, Y. Li, J. Li, Modelling and Simulation in Materials Science and Engineering 18 (2010) 085008

[13] D. Cereceda, J.M. Perlado, S. Queyreau, A. Stukowski, L. Ventelon, M.C Marinica, J. Marian, Model. Simul. Materials Science and Engineering, submitted for publication. 